\title{
HEEGAARD SURFACES IN HAKEN 3-MANIFOLDS
}

\author{
KLAUS JOHANNSON
}

The purpose of this note is to announce, for the case of Haken 3-manifolds, a complete solution of Waldhausen's conjecture concerning finiteness for Heegaard surfaces.

It is a classical result due to Moise [Moi] that all compact (orientable) 3-manifolds $N$ have finite triangulations. Closely related to triangulations are Heegaard graphs, i.e., those finite, possibly disconnected, graphs $\Gamma$ in $N$ with $\Gamma \cap \partial N=\partial \Gamma$ and the property that $(N-U(\Gamma))^{-}$is a handlebody (here $U(.$.$) denotes$ the regular neighborhood in $N$ ). Indeed, the 1-skeleton of any triangulation of $N$ is a Heegaard graph-but of course not vice versa. A surface $F$ in $N$ is a Heegaard surface, provided there is a Heegaard graph $\Gamma$ in $N$ such that $F$ is isotopic in $N$ to $\partial U(\Gamma \cup \partial N)-\partial N$. If $F$ is a Heegaard surface, the pair $(N, F)$ is called a Heegaard splitting for $N$. Heegaard splittings are independent of triangulations, and yet closely connected to them.This suggests we should use them for a classification of 3-manifolds. In this context Reidemeister [Re] and Singer [Si] showed that the existence of a common subdivision for any two triangulations implies that Heegaard surfaces in 3-manifolds are stably isotopic, i.e., isotopic modulo finite connected sums with the standard torus in $S^{3}$. Based on this observation, Waldhausen [Wa1] proved that any two Heegaard surfaces of $S^{3}$ are isotopic iff they have the same genus. Using different methods, this result has been extended to Heegaard surfaces of lens spaces [Bo, $\mathrm{BO}]$ and minimal (see below) Heegaard surfaces of the 3-torus [FH]. However, in [BGM] explicit examples of irreducible 3-manifolds are constructed with at least two nonhomeomorphic Heegaard splittings. In fact, it is now clear [BRZ] that uniqueness of Heegaard surfaces fails drastically and is a rather special phenomenon for 3-manifolds. In

Received by the editors July 26, 1989 and, in revised form, October 20, 1989. 1980 Mathematics Subject Classification (1985 Revision). Primary 57M99.

Key words and phrases. Haken 3-manifolds, $n$-relator 3-manifolds, handlebodies, Heegaard surfaces, essential surfaces, Dehn twists.

Supported in part by NSF Grant DMS-8803256. 
this context Waldhausen raised in 1978 the following problem for closed 3-manifolds $N$ :

Waldhausen Conjecture. [Wa2] Show that $N$ has only finitely many isomorphism classes (or even isotopy classes) of minimal Heegaard splittings.

Given any of these, give a procedure to obtain the others.

Here a Heegaard splitting $(M, S)$ is called minimal if the Euler characteristic of $S$ is as large as possible, and it is called irreducible if it cannot be obtained from some other Heegaard splitting by adding a trivial 1-handle (= connected sum with the standard torus in $S^{3}$ ). This choice of notation differs from that of [Wa2] where irreducible Heegaard splittings are called minimal-partly due to the then common (but wrong) expectation that these two notions are the same anyway. But the above conjecture of Waldhausen is known to be false for irreducible Heegaard surfaces since, according to Casson and Gordon (unpublished), there are closed 3-manifolds which contain irreducible Heegaard surfaces of any genus. It is further not difficult to construct 3-manifolds which contain infinitely many nonisotopic, minimal Heegaard splittings. In the following we are concerned with the sets $\mathscr{H}(N, m)$ of all (isotopy classes of) Heegaard surfaces $F$ in $N$ with $-\chi(F)=m$. Given a Heegaard surface $F \in \mathscr{H}(N, m)$, let us further denote by $\mathscr{K}(F)$ the set of all maximal systems of simple closed, noncontractible and pairwise nonparallel curves in $F$ which bound a system of discs on one side of $F$.

Because of the early difficulties with Heegaard surfaces, Haken [Ha1, Ha2] suggested to approach the classification problem via a study of the more accessible class of essential surfaces in 3manifolds, and to exploit Heegaard surfaces (or better Heegaard diagrams) for this purpose. Here an orientable surface is essential if it does not bound a ball and cannot be ( $\partial$-)compressed along a disc, and $\mathscr{S}(N, m)$ denotes the set of all isotopy classes of essential surfaces $S$ in the 3-manifold $N$ with $\chi(S)=-m$. Essential surfaces, different from the 2-sphere, exist in many 3-manifolds, notably all 3-manifolds with infinite first integer homology and so, e.g., for all 3-manifolds which have a boundary component different from a 2-sphere. Three-manifolds which are $(\partial$-)irreducible and contain essential surfaces are today called Haken 3-manifolds since Haken obtained basic results of far-reaching consequences concerning essential surfaces in 3-manifolds. Specifically, he asso- 
ciated (in a slightly different terminology) to any $F \in \mathscr{H}(N, m)$ and to any $k \in \mathscr{K}(F)$ a certain polynomial $f=f_{(F, k)}$, and showed that every essential surface $S$ in a simple 3-manifold $N$ can be isotoped so that afterwards $(1) \#(S \cap F) \leq f(\chi S)$ and (2) $S \cap F \subset U(k)$. Here a Haken 3-manifold is simple if it contains no essential annuli and tori. To appreciate the significance of this theorem, notice that, e.g., the finiteness of the sets $\mathscr{S}(N, m)$ is an immediate consequence for all simple 3-manifolds $N$. Using the work of Hemion, together with the characteristic submanifold theory, this finiteness theorem ultimately yields, among other things, a solution of the homeomorphism problem for Haken 3manifolds [Ha2, He]. From a numerical point of view, the above result of Haken is less satisfying, for a computation of the polynomials $f_{(F, k)}$ requires the determination of a positive basis of certain systems of linear equations which are in general also rather large. Using different methods however, Haken [Ha3] managed to demonstrate that a great improvement of the relevant estimate is possible in the case of 2-spheres. Indeed, he showed the existence of essential 2-spheres in 3-manifolds intersecting a given Heegaard surface in one curve only, provided the 3-manifold contains an essential 2-sphere at all. In [Och, $\mathrm{Ko}, \mathrm{CH}$ ] similar results have been obtained in the special cases of projective planes, as well as nonseparating tori and Klein bottles in orientable and nonorientable 3-manifolds of Heegaard genus 2; but there is certainly no reason to always expect essential surfaces intersecting a given Heegaard surface in just one curve. At this point notice that Haken's above 2 -sphere result is not only a result about 2 -spheres, but also a result about Heegaard surfaces since, according to his result, the minimal number of intersections of 2-spheres with Heegaard surfaces does not depend on the actual choice of the Heegaard surface at all. Here is a generalization of this aspect of Haken's theorem.

Theorem 1. To any Haken 3-manifold $N$, there is associated a polynomial function $g: \mathbf{N} \times \mathbf{N} \rightarrow \mathbf{N}$ and, to every Heegaard surface $F$ in $N$, there is associated a finite and constructible subset $\mathscr{L}(F) \subset \mathscr{K}(F)$ with the following property: Every essential surface $S$ in $N$ can be isotoped so that afterwards

(1) $h S \cap F \subset U(k)$, for some $k \in \mathscr{L}(F)$, and

(2) $\#(h S \cap F) \leq g(\chi S, \chi F)$,

for some finite product $h$ of Dehn twists along essential tori in $N$. 
There is also a version of this theorem for normal surfaces in 3manifolds-including essential 2-spheres. The method of proof is different from Haken's approach and so represents an independent basis for Haken's normal surface theory. In contrast to Haken's theory, however, there is a simple formula for the function $g$ which avoids the computation of positive basis at all. A computation of this function in special cases yields Haken's 2-sphere result as well as the forementioned extensions given in [Och, Ko].

Technically, Theorem 1 may be read as a result about essential surfaces in $n$-relator 3-manifolds, where, by definition, an $n$ relator 3-manifold, notation $M^{+}(k)$, is given by some handlebody $M$ with $n$ 2-handles attached along a system $k$ of $n$ curves in $\partial M$. The theorem may be interpreted as saying that the study of essential surfaces in $n$-relator 3-manifolds $M^{+}(k)$ (and so in all Haken 3-manifolds) can be reduced to the study of essential surfaces in the relative handlebody $(M, k)$. Now, essential surfaces in relative handlebodies turn out to be rather accessible. In fact, a number of results about 3-manifolds have their counterparts for relative handlebodies - but are much easier to prove for the latter. Specifically, we can give a computational form of the Poincare map for relative handlebodies which allows the computation of the Thurston norm for relative handlebodies, and so, by Theorem 1, for Haken 3-manifolds. Moreover, we can show that incompressibility for surfaces in 3-manifolds can be decided in polynomial time (w.r.t. the Euler characteristic). Theorem 1 tells us that in any $n$-relator 3-manifold $M^{+}(k)$ the intersection of essential surfaces with the attached 2-handles is limited. This intersection is not necessarily empty, i.e., 2-handles and surfaces are not necessarily disjoint, but the underlying obstruction to disjointness can be understood for essential surfaces. Indeed, there are two simple geometric operations for surfaces, which we call "annulus-" and "torus-modifications," and which turn (modulo handleslides) essential surfaces in $\mathrm{M}^{+}(k)$ into surfaces avoiding at least one of the 2-handles, without decreasing the Euler characteristic of the surface and without leaving its homology class. A particular interesting case occurs when $\mathrm{M}^{+}(k)$ is a one-relator 3-manifold. In this particular case it also follows that any essential disc in an irreducible one-relator 3-manifolds can be isotoped out of the 2-handle (improving results of [ $\mathrm{Pr}, \mathrm{Ja}, \mathrm{Joh} 2, \mathrm{Sch}]$ and others). The cocore of the 2-handle is a proper arc $t$ in the 3-manifold 
$N=M^{+}(k)$ whose complement is a handlebody, and such an arc is called a Heegaard string. Since splitting $N$ along an essential surface avoiding $t$ is again a one-relator 3-manifold, the above result yields inductively a hierarchy of $N$ which is disjoint to $t$. Studying arcs in the complement of hierarchies, we obtain the following:

Proposition 2. Let $t$ be a Heegaard string in a 3-manifold $M$ which can be deformed (modulo endpoints) into $\partial M$. Then $M$ is a handlebody and $t$ is unknotted.

This result, taken together with the characteristic submanifold theory [Joh1] (see also [JS]), yields the following result about the relevant mapping class groups.

Proposition 3. Let $M^{+}(k)$ be a one-relator 3-manifold which is irreducible and $\partial$-irreducible. Let $\varphi: \pi_{0} \operatorname{Diff}(M, k) \rightarrow \pi_{0} \operatorname{Diff} M^{+}(k)$ be given by extension. Then there is a, possibly nonorientable, surface $S$ with $\pi_{1}(S) \cong \operatorname{kern} \varphi$. In addition, $S$ is bounded if $M^{+}(k)$ is not the I-bundle over some surface, and it is the disc if $M^{+}(k)$ is a simple 3-manifold.

As a consequence we obtain that the mapping class group of a simple one-relator 3-manifold is a subgroup of a dihedral group, provided its minimal Heegaard splitting is unique (up to isotopy).

Theorem 1 may also be read as a statement about Heegaard graphs in Haken 3-manifolds. Indeed, it says that modulo edgeslides and Dehn twists along essential tori there is, for any fixed essential surface $S$ in $N$, a constant $c_{S}$ such that $\#(\Gamma \cap S) \leq c_{S}$, for all Heegaard graphs $\Gamma$ in $N$. Now, according to [Ha2], every essential surface can be taken as the beginning of a hierarchy of essential surfaces, and a complexity which measures the complexity of embedding types of Heegaard graphs with respect to hierarchies may be defined. It turns out that whenever this complexity increases over a certain constant (depending on $N$ alone), one of a small list of simple geometric objects emerges, which then can be analyzed directly. The formal result of this analysis reads as follows:

Theorem 4. Let $N$ be a Haken 3-manifold (closed or not), and let $\mathscr{A}(N)$ denote the subgroup of the mapping class group of $N$ generated by all Dehn twists along tori. Then, for every integer $m$, the quotient $\mathscr{H}(N, m) / \mathscr{A}(N)$ is finite and constructible. 
(J. Hass announced the finiteness for minimal Heegaard surfaces of closed hyperbolic 3-manifolds of Heegaard genus two.)

It follows in particular that the Heegaard genus can be computed for every Haken 3-manifold $N$. Notice further that the previous finiteness result is formally similar to Haken's theorem that there are, modulo Dehn twists along annuli and tori, only finitely many (isotopy classes of) essential surfaces of given Euler characteristic in $N$, the only difference being that Dehn twists along annuli apparently do not affect Heegaard surfaces. All this fits very well with the known fact [Joh1] that the mapping class group of Haken 3-manifolds contains the group, generated by all Dehn twists along essential annuli and tori, as a subgroup of finite index. The proof of the above theorem, however, does not use this very fact about the mapping class group, and so the characteristic submanifold shows up as the geometric obstruction for yet another important property of Haken 3-manifolds. From a computational point of view, it may be interesting to note that, for closed Haken 3-manifolds $N$, the counting function, given by $m \mapsto \# \mathscr{S}(N, m) / \mathscr{A}(N)$, has polynomial growth. Moreover, recall that, by the Reidemeister-Singer theorem, any two Heegaard surfaces $F, G$ in a 3-manifold $N$ are stably equivalent, and so the minimal number of stabilizations may be taken as a distance between $F$ and $G$. This distance turns the set of Heegaard surfaces in $N$ into a metric space. Given a metric space $X$, let $\operatorname{diam} X$ denote the largest distance in $X$.

Theorem 5. Let $N$ be a Haken 3-manifold (closed or not). Then the assignment $m \mapsto \operatorname{diam} \mathscr{H}(N, m) / \mathscr{A}(N)$ defines an arithmetic function of polynomial growth.

This result is based on Theorem 4 and a new proof of the Reidemeister-Singer theorem [Joh4] which yields an estimate for the above distance in terms of winding numbers for Heegaard graphs as introduced in [Joh4]. In contrast to this result, it can be shown (extending a result of [BRZ]) that the total numbers of minimal Heegaard surfaces in Seifert fiber spaces over the disc are subdominated by some arithmetic function of exponential growth with respect to the number of exceptional fibers.

Combining the above results with the classification of homotopy equivalences in Haken 3-manifolds with boundary [Joh1], we finally also obtain an affirmative answer to a question of Zieschang. Indeed, given any handlebody $M$ and any simple closed curve 
$k$ in $\partial M$, denote by $[k]$ the set of all isotopy classes of simple closed curves in $\partial M$ which are homotopic in $M$ to $k$. Then we have the following:

Theorem 6. The set $[k] / \pi_{0}$ Diff $M$ is finite, for all handlebodies $M$ and all simple closed curves $k$ in $\partial M$.

This result can be extended to systems of curves as well, but then the statement is technically more complicated since the possibility of handleslides has to be taken into account. Related results for the case of genus two handlebodies can be found in [Zie]. As another application of the classification of homotopy equivalences and our solution of the Waldhausen conjecture, we obtain that, modulo finitely many and constructible choices, the presentations of isomorphic fundamental groups of given Haken 3-manifolds (including knot groups) can be obtained by a sequence of Tietzetransformation, involving no stabilization at all.

Further discussion and complete proofs of the above results can be found in [Joh3].

\section{REFERENCES}

[BGM] J. S. Birman, F. Gonzàlez-Acuña, J. M. Montesinos, Heegaard splittings of prime manifolds are not unique, Mich. Math. J. 23 (1976), 97-103.

[BRZ] M. Boileau, M. Rost, and H. Zieschang. Décompositions de Heegaard des extérieurs des noeds toriques et des variétés de Seifert associées, C. R. Acad. Sci. Paris Sér. I Math. 302 (1986), 661-664.

[Bo] F. Bonahon, Difféotopies des espaces lenticulaires, Topology 22 (1983), 305-314.

[BO] F. Bonahon and J.-P. Otal, Scindement de Heegaard des espaces lenticulaires, C. R. Acad. Sci. Paris Sér. I Math. 294 (1982), 585-587.

[CH] I. Cardona and W. Heil, Essential tori and Klein bottles in non-orientable 3-manifolds of genus two, Geometry and Topology, (G. M. Rassias \& G. M. Stratopouloos, eds.) World Scientific Publ. Co., Singapore, 1989, pp. 31-57.

[FH] C. Frohman and J. Hass, Unstable minimal surfaces and Heegaard splittings, Invent. Math. 95 (1989), 529-540.

[Ha1] W. Haken, Theorie der Normalflächen, Acta Math. 105 (1961), 245-375.

[Ha2] - Über das Homöomorphieproblem der 3-Mannigfaltigkeiten I, Math. Z. 80 (1962), 89-120.

[Ha3] _ Some results on surfaces in 3-manifolds, Studies in Modern Topology, no. 5, Math. Assoc. Amer. (P. J. Hilton, ed.) 1968, 39-98.

[He] G. Hemion, On the classification of homeomorphisms of 2-manifolds and the classification of 3-manifolds, Acta Math. 142 (1979), 123-155.

[Ja] W. Jaco, Adding a 2-handle to a 3-manifold: An application to property $R$, Proc. Amer. Math. Soc. 92 (1984), 288-292. 
[JS] W. Jaco and P. B.Shalen, Seifert fibre spaces in 3-manifolds, Memoirs of the Amer. Math. Soc. 21, no. 220, (1979).

[Joh1] K. Johannson, Homotopy equivalences of 3-manifolds with boundaries, Lecture Notes in Math., vol. 761, Springer-Verlag, Berlin, (1978).

[Joh2] _-, On surfaces in one-relator 3-manifolds, London Math. Soc. Lecture Note Ser., vol. 112, Cambridge Univ. Press, New York, (1986), 157-192.

[Joh3] - Computations in 3-manifolds, preprint.

[Joh4] K. Johannson, On the Reidemeister-Singer theorem, preprint.

[Ko] T. Kobayashi, Non-separating essential tori in 3-manifolds, J. Math. Soc. Japan 36 (1984), 11-22.

[Moi] E. E. Moise, The triangulation theorem and Hauptvermutung, Ann. of Math. 56 (1952), 96-114.

[Och] M. Ochiai, On Haken's theorem and its extensions, Osaka J. Math. 20 (1983), 461-488.

[Pr] J. H. Przytycki, Incompressibility of surfaces after Dehn surgery, Michigan Math. J. 30 (1983), 289-308.

[Re] K. Reidemeister, Zur dreidimensionalen Topologie, Abh. Math. Sem. Univ. Hamburg 9 (1936), 189-194.

[Si] J. Singer, Three-dimensional manifolds and their Heegaard-diagrams, Trans. Amer. Math. Soc. 35 (1933), 88-111.

[Sch] M. Scharlemann, The Thurston norm and 2-handle addition, Proc. Amer. Math. Soc. 100 (1987), 362-366.

[Wa1] F. Waldhausen, Heegaard Zerlegungen der 3-Sphäre, Topology 7 (1968), 195-203.

[Wa2] _ Some problems on 3-manifolds, Proc. Sympos. Pure Math., vol. 32, Amer. Math. Soc., Providence, RI, (1978), 313-322.

[Zie] H. Zieschang, Classification of simple systems of paths on a solid pretzel of genus 2, Soviet. Math. Dokl., vol. 4, Amer. Math. Soc., Providence, RI, 1963, 1460-1463. Transl. of Doclady Acad. Sci. USSR 152 (1963), 841-844.

Department of Mathematics, University of Tennessee, Knoxville, TENNESSEE 37966-1300 